\title{
Human Papillomavirus (HPV) Infections and the Importance of HPV Vaccination
}

\author{
Chia-ching J. Wang ${ }^{1} \cdot$ Joel M. Palefsky ${ }^{2}$
}

Published online: 26 March 2015

(C) Springer International Publishing AG 2015

\begin{abstract}
HPV persistence is necessary for the development of anogenital cancer. Studies show that cervical and anal HPV infections in women and in men who have sex with men are common. Clearance of HPV infection is similarly common; few individuals show persistence unless they are HIV-infected. HIV strongly influences the development of cervical and anal cancer, as well as their premalignant counterparts. Women with cervical and vulvar HPV-associated lesions have higher rates of anal cancer than the general population. HPV also plays an important role in pathogenesis of head and neck cancers, particularly oropharyngeal cancer. Two commercially available HPV vaccines have been proven to be safe and efficacious against cervical HPV16/18 infections and associated precancerous lesions; one of these has also been shown to prevent HPV16/18-associated anal lesions. The FDA has also just approved a new nonavalent HPV vaccine. HPV vaccines
\end{abstract}

Chia-ching J. Wang holds a MD degree, SUNY-Stony Brook. Joel M. Palefsky holds a MD degree, McGill University (Montreal).

This article is part of the Topical Collection on Infectious Disease Epidemiology

Joel M. Palefsky

joel.palefsky@ucsf.edu

Chia-ching J. Wang

chia-ching.wang@ucsf.edu

1 Division of Hematology/Oncology, Helen Diller Comprehensive Cancer Center, University of California, San Francisco, 505 Parnassus Avenue, Box M1270, San Francisco, CA 94143, USA

2 Division of Infectious Diseases, University of California, San Francisco, 513 Parnassus Ave, Med Sci Room 420E, Box 0654, San Francisco, CA 94143, USA will play an important role in prevention of HPV-associated cancers.

Keywords HPV · HPV vaccination · Cervical cancer · Anal cancer $\cdot \mathrm{HIV} \cdot$ Immunosuppression

\section{Introduction}

HPV infections are almost exclusively acquired from sexual exposure by entering the skin and mucous membranes of the mouth, anus, penis, and female reproductive tract. Concordance of HPV infection between sexual partners is variable and ranges from 40 to $60 \%$, which may be affected by length of sexual relationship, frequency of intercourse, condom use, and number of lifetime sexual partners [1,2]. Once HPV infects one sex partner, it is quickly transmitted to another [3]. In a 12-month period, the probability for men to acquire a new genital HPV infection is estimated to be $0.29-0.39$, which is similar to previous estimates for women [4, 5]. Additionally, the overall transmission rate from one heterosexual partner to the other over a 6-month period is estimated to be 3.7 cases per 100 person-months [6].

HPVs are small, non-enveloped DNA viruses and there are over 150 types. HPV genotypes 16 and 18 are responsible for approximately $70 \%$ of cervical cancers, with the remaining $30 \%$ caused by other carcinogenic HPV types [7]. HPV16 is the most carcinogenic type in terms of numbers of cases of cervical intraepithelial neoplasia (CIN) or cervical cancer [8]. Low-risk genotypes 6 and 11 cause most cases of genital warts. There is conflicting evidence regarding whether any type of oncogenic HPV (especially HPV16) persists longer than others [9] in the absence of development of CIN3+. However, it is clear that viral type is a major predictor of risk of CIN3+ [10]. 
HPV infection accounts for approximately $5.2 \%$ of human cancer burden worldwide, including the cancers of the anus, genital tract, and oropharynx $[11,12]$. Each year, approximately 500,000 women are diagnosed with invasive cervical cancer worldwide and 250,000 women die from it, mainly in developing countries [13]. The incidence of anal cancer is low among men in the general population but is considerably increased among men who have sex with men (MSM). Among HIVinfected MSM, the incidence rates are even higher [14]. The risk of anal cancer has continued to increase among HIVinfected MSM since the introduction of highly active antiretroviral therapy (HAART) [15], and it is possible that this trend may continue with the aging of the HIV-infected population. Currently, rates of anal cancer in HIV-infected MSM are higher than cervical cancer reported anywhere in the world [16].

\section{Biology of HPV}

In normal squamous epithelium of the cervix, the basal layer is the area of active cell division. After division, the cells migrate up from the basal layer and no longer progress through the cell cycle and become terminally differentiated keratinocytes. In the anus, intraepithelial lesions generally arise in the transition zone, a region that extends from the squamocolumnar junction with the rectal columnar mucosa distally to the dentate line. In this area of transition, there is active transition from columnar epithelium to squamous epithelium through the process of squamous metaplasia. Upon entry into either cervical or anal epithelium at areas of microtrauma, HPV targets actively proliferating basal cells. Most viral genome replication occurs after the epithelial cells migrate away from the basal layer. Since the epithelial cells have stopped dividing at this stage, the viral copy number per cell greatly increases, as do levels of viral gene expression. Typical HPV-induced histopathologic changes characteristic of low-grade lesions reflect active viral replication. These include koilocytosis, multinucleation, and nuclear enlargement and are due to the assembly of the viral particles in the upper epithelial layers. The epithelium is then shed, and infectious HPV virions are released, which can then infect a new host.

In contrast, HPV-induced histopathologic changes associated with high-grade, precancer lesions reflect the activity of HPV oncoproteins such as E6 and E7. E6 binds to and degrades tumor-suppressor protein p53 in the host cell, inhibiting apoptosis and cell cycle arrest and leading to cellular proliferation [17]. The E7 protein is involved with cell immortalization through the retinoblastoma $(\mathrm{Rb})$ proteins. When E7 binds Rb, the Rb-E7 complex is degraded and the cell proceeds unregulated through the $\mathrm{S}$ phase [18]. Currently, it is believed that low-risk HPV types do not cause malignancy due to weaker binding of their E6 and E7 to their target proteins, differences in promoter positioning and regulation, and pattern of mRNA splicing compared to E6 and E7 from the high-risk HPV types [19, 20].

Both the anus and cervix are highly susceptible to HPV infection, and a spectrum of pathologic changes may occur as a result of HPV infection. The terminology for HPVassociated squamous lesions of the lower anogenital tract has a long history marked by various diagnostic terms derived from multiple specialties. The Lower Anogenital Squamous Terminology (LAST) project aimed to create a histopathologic nomenclature system that reflects current knowledge of HPV biology. Current data support the two-tiered system of low-grade squamous intraepithelial lesions (LSIL) and highgrade squamous intraepithelial lesion (HSIL) [21••], which may be further qualified with the appropriate -IN terminology for specific location. Therefore, LSIL includes condyloma and CIN1 or anal intraepithelial neoplasia (AIN) 1 and is not considered to be precancerous. In contrast, HSIL includes p16positive CIN2 and AIN2, along with CIN3 and AIN3. HSIL are considered to be the true cancer precursors $[21 \bullet \bullet]$.

\section{Cervical HPV Infection}

In the 2011 National Health and Nutrition Examination Survey (NHANES) in the USA, cervicovaginal specimens from more than 4000 women were included in a cross-sectional analysis. The overall prevalence of 37 different types of HPV was $42.5 \%$, which represents approximately 40 million infections [22]. The lifetime risk of having genital HPV infection is even higher, making HPV the most common viral sexually transmitted infection (STI).

HPV infection often occurs shortly after initiation of sexual activity. In one study of college-aged women, the cumulative incidence of any HPV infection at 1 year after sexual debut was $28.5 \%$ and increased to almost $50 \%$ by 3 years [23]. HPV is usually transmitted through vaginal or anal intercourse, but it can occur through oral-genital or genitalgenital contact as well. In young women who reported no previous sexual intercourse but had genital-genital or genital-oral contact, the 24-month cumulative incidence of infection was $7.9 \%(95 \% \mathrm{CI}, 3.5-17.1)$ [23]. The risk of genital HPV infection among women who report only sexual experience with other women was reported to be similar to their heterosexual counterparts [24].

Despite their high prevalence, most cervical HPV infections are transient and asymptomatic, with about $70 \%$ of new infections resolving within a year [25]. Young women are more likely to clear infections than older women [26]. A negative oncogenic HPV test implies low risk of CIN3+, and a positive oncogenic HPV test implies a higher risk [27]. In one cohort, women who tested positive for an oncogenic HPV test at baseline and 2 years later had an absolute risk of CIN3+ at 12 years of $19.3 \%$ [27]. For HPV16, the risks of CIN3+ at 3, 
5 , and 12 years of follow-up among women with two positive tests were $8.9 \%(2.5-14.9 \%), 23.8 \%(14.1-32.4 \%)$, and $47.4 \%$ (34.9-57.5\%), respectively [28]. Among women who do not clear their infection, studies report variable rates of progression to CIN2/3 ranging from 8 to $28 \%$ [29-31]. Fortunately, the transition to cancer usually takes years or decades; the peak incidence of HPV infection occurs at about age 20, the peak incidence/detection of CIN3 occurs at about age 30 , and the peak incidence of cancer occurs in the $40 \mathrm{~s}$ [32]. For women with oncogenic HPV infection, smoking has been implicated as a significant risk factor for CIN3/cervical cancer [33].

\section{Oral HPV Infection}

The epidemiology and natural history of oral HPV infection have not been well established. It appears that oral HPV prevalence is substantially lower than genital HPV infection, with the prevalence of any HPV infection being $4.5 \%$ in a pooled analysis [34]. In the NHANES data, prevalence of HPV had a striking bimodal pattern with age among men and was significantly higher among men than women. This is also consistent with higher rates of HPV-positive oropharyngeal cancer among individuals aged 50 to 64 years and among men. Notably, prevalence was as high as $20 \%$ among those with more than 20 lifetime sexual partners or among current smokers of more than 20 cigarettes per day [35].

Head and neck cancer (HNC) mainly includes squamous cell carcinomas occurring in the oral cavity, pharynx, and larynx. The role of high-risk HPV infection in the etiology of HNC has gradually emerged during the past two decades. During this period, an increase in incidence rates of oropharyngeal cancer has been reported in men in high-income countries despite decreasing rates of other tobacco-associated cancers including oral cancer [36]. In addition, the fraction of HPV-positive oropharyngeal cancer increased in the same period in the USA and Europe, whereas no clear change was observed for other $\mathrm{HNC}[36,37]$. In a recent meta-analysis, regardless of the type of marker that had been used for detection, the probability of a non-oropharyngeal HNC being attributable to HPV is at least fivefold lower than that for oropharyngeal cancer [38]. However, data on HPV in other less common HNCs are very limited and essentially negative. Additional studies are also needed to establish the way HPV infection and tobacco may interact in non-oropharyngeal HNC.

\section{Anal HPV Infection}

In cross-sectional studies, anal HPV infection is almost universal among HIV-infected MSM, with reported prevalence estimates between 87 and $98 \%$ [39, 40]. In one study, HIVinfected and HIV-negative MSM both had high rates of HPV clearance, with almost $75 \%$ of HPV infection cleared within 1 year [40]. Interestingly, HPV infection rates seem to stay constant in men, independent of age, as opposed to women, among whom the prevalence of anal HPV is highest during 18-24 years of age and then decreases until middle age, after which it generally remains steady [3]. A prospective cohort study to assess the natural history of anal HPV infection in MSM in the HAART era showed the incidence of any anal HPV infection and oncogenic anal HPV infection was 21.3/ 100 person-years and 13.3/100 person-years, respectively [41•]. These were greater than the reported incidence of other STIs among HIV-infected MSM [41•]. Twenty percent of these men with an incident HPV infection also had more than one new HPV type detected during follow-up [42]; many had multiple oncogenic types which might be associated with a greater likelihood of AIN2/3 [43].

Genital HPV infection, primarily penile infection, is common among sexually active heterosexual men and MSM, but there is also considerable variation in prevalence and incidence, depending on age, country, and region. In an analysis of 1732 males aged 16-24 years old in the placebo arm of a quadrivalent HPV vaccine trial, the acquisition of genital HPV $6,11,16$, and/or 18 in males was common and rates of HPV DNA detection were highest in men from Africa (17.2 cases per 100 person-years), while Asia-Pacific had the lowest incidence (3.2 cases per 100 person-years) [44]. The researchers also found that it took on average 6 months for genital HPV infections to clear, and most of these infections were detected when there were no visible external genital warts [44]. In Australia, when $65 \%$ of women eligible for HPV vaccines were vaccinated, proportionally fewer heterosexual men were diagnosed with genital warts during the vaccine period, consistent with herd immunity, and this effect was more pronounced in young men [45•]. Given these facts, HPV vaccination of women may reduce infection in men and reduce the overall burden of HPV-associated disease in the community. Vaccination of males will also reduce the incidence of genital HPV infection in males and could potentially produce herd immunity that benefits their male and female sexual partners.

\section{HIV and Immunosuppression}

The immune system plays a major role in persistence of HPV infections, and the oncogenic potential of HPV may be enhanced by immunosuppression (Fig. 1). Historically, most of the experience with immunocompromise and HPV-related diseases has been in the setting of solid organ transplant [47-49]. Organ transplant recipients have a high risk of developing other malignancies, particularly cancers that have been linked to viral infections, because of lifelong 
Fig. 1 Anal cancer incidence in the general US population (1980 2005). Reprinted with permission from [46] by permission of Oxford University Press and the National Cancer Institute
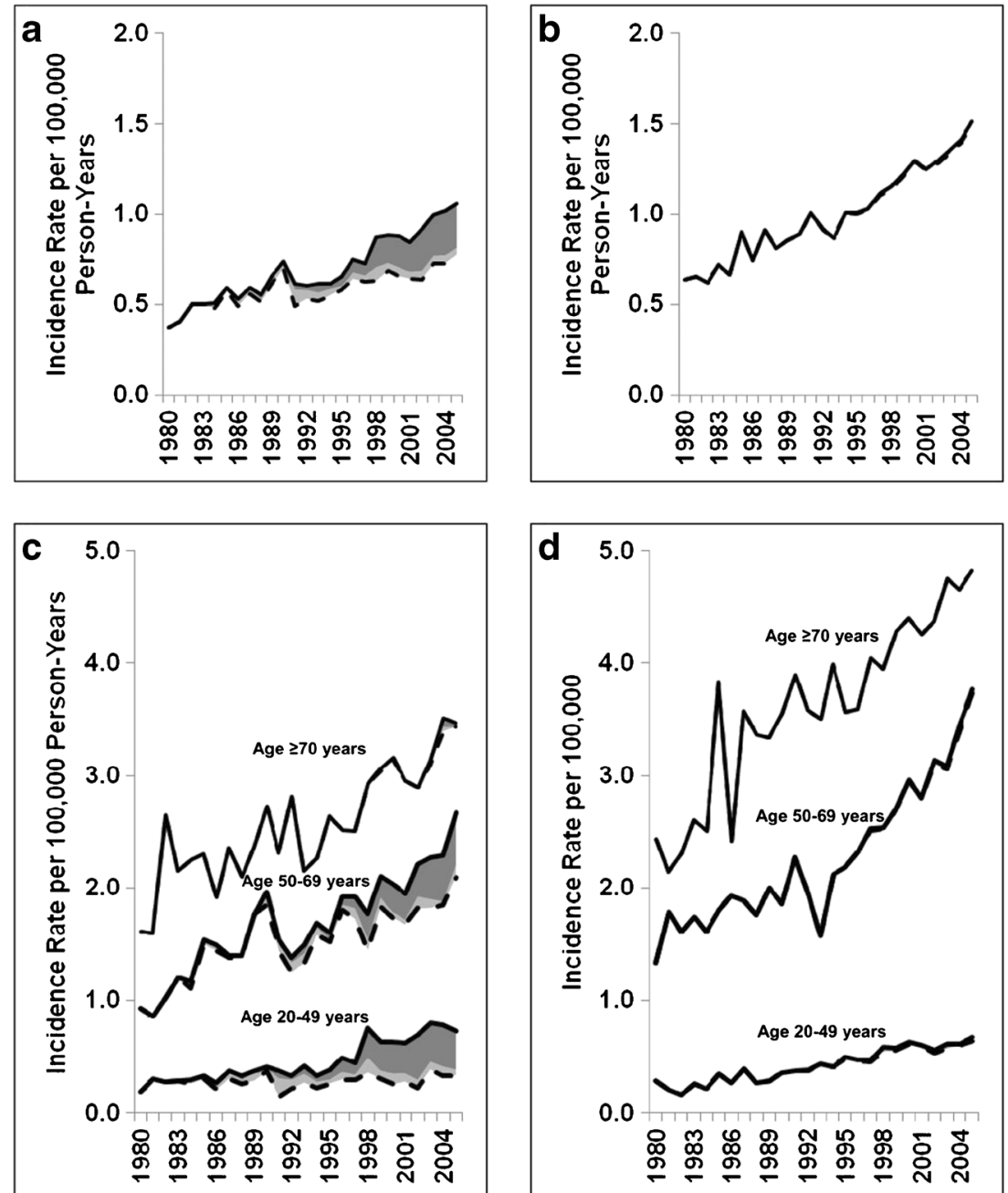

immunosuppressive therapy. More recently, HIV infection has emerged as the most common cause of immunocompromise, with a meta-analysis showing that the pattern of increased risk of cancers is similar in people with HIV/AIDS and in transplant recipients [50]. This suggests immune deficiency is an important contributor to the increased risk of cancer.

Women with HIV have high rates of cervical HPV infection, and their risks of cervical precancer and cancer are increased several-fold compared with those in the general population [51-54]. With lower CD4 counts and higher plasma HIV RNA levels, the prevalence, incident detection, and persistence of HPV and CIN among HIV-infected women increase [54]. Detection of incident HPV infection and CIN is related to plasma HIV levels even when CD4 count is low [55], perhaps reflecting the detrimental effects of HIV on host immunity beyond total CD4 T cells [56].

It is not yet clear if local HIV RNA levels in the genital tract are associated with cervical HPV infection and neoplasia. One prior study reported that high cell-free genital tract HIV RNA levels were related to HPV detection [57]. Another small cross-sectional study showed CIN was not related to cervical
HIV RNA detection [58]. Recently, in the Women's Interagency HIV Study (WIHS) cohort, a nested case-control study showed risk of CIN2/3 was associated with genital tract HIV RNA levels $(\mathrm{OR}=2.96, p=0.03)$ [59]. However, this association became insignificant after adjustment for plasma HIV RNA levels. Genital tract HIV RNA levels were not associated with the prevalence of any HPV or oncogenic HPV [59]. However, the incident detection of any HPV $(p=0.02)$ and persistence of oncogenic HPV $(p=0.04)$ were associated with genital tract HIV RNA levels, after controlling plasma HIV RNA levels [59]. These data suggest that genital tract HIV RNA levels are not a significant independent risk factor for cervical precancer in HIV-infected women, but they may influence HPV infection.

The prevalence of anal HPV infection and AIN is high among HIV-infected men and women. In a study of 357 HIV-infected MSM, over $80 \%$ had anal dysplasia and $95 \%$ had HPV infection [60]. In the San Francisco/Oakland Cancer Registry, significant increases in the population-based incidence rates of carcinoma in situ among men were observed between 2000 and 2009 [61], reflecting the high incidence of 
HIV infection in the preceding 20 years and the fact that these men are also at high risk of HPV infections and AIN. Among $200 \mathrm{HIV}$-infected or high-risk HIV-negative women for whom there were concurrent anal and cervical HPV data, anal HPV was more common than cervical HPV in both HIV-infected ( $79 \%$ anal vs $53 \%$ cervical) and HIV-negative women (43\% anal vs $24 \%$ cervical) [62]. In another study, anal HPV infection was more prevalent and diverse than cervical HPV infection, and anal cytologic abnormalities were as prevalent as cervical cytologic abnormalities [63•]. HIV infection has been shown to be a strong risk factor for concomitant anal and cervical HPV infection [64]. It has already been projected that screening HIV-infected MSM for AIN2/3 has been shown to prolong quality-adjusted life expectancy [65]. HIV-infected women, particularly those women with low CD4 cell counts, may also be good candidates for HPV screening and monitoring for both cervical and anal disease, as well as those with prior cervical or vulvar cancer or CIN/VIN3.

\section{HPV Vaccination}

Although there is no known cure for persistent HPV infection, two vaccines are currently available that protect against HPV types 16 and 18: the bivalent vaccine Cervarix ${ }^{\mathrm{TM}}$ (GlaxoSmithKline) and the quadrivalent (qHPV) vaccine

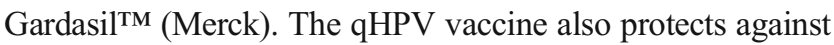
HPV types 6 and 11. Both of these vaccines are produced using recombinant technology by inserting the L1 gene into a host (either yeast or baculovirus), which then produces L1 proteins that self-assemble into virus-like particles. Because these particles do not contain viral DNA, they are neither infectious nor oncogenic [66]. Both vaccines are given in a three-dose schedule as intramuscular injections. The complete series of three injections must be administered prior to sexual debut for these vaccines to be most effective [67].

HPV vaccination has emerged as an important element of cancer prevention. The bivalent HPV vaccine was evaluated in a phase III trial of girls and women aged 15-25 years; its efficacy against CIN2+ was $94.9 \%$ [68]. The efficacy against 6- and 12-month persistent HPV 16 or 18 cervical infections in the per-protocol cohort was $94.3 \%(96.1 \% \mathrm{CI}=91.5-96.3)$ and $91.4 \%$ (96.1 \% CI=86.1-95.0), respectively [69]. Among participants who were DNA-positive to one vaccine HPV type, the bivalent vaccine also had high efficacy $(90 \%)$ for prevention of CIN2/3 associated with the type for which a female was DNA-negative at enrollment [70]. The efficacy of the $\mathrm{qHPV}$ vaccine for prevention of HPV-related precancerous lesions and disease is supported by studies among females. In three trials, qHPV had high efficacy ( $>98 \%$ ) for prevention of HPV 6-, 11-, 16-, and 18-related CIN2/3 or adenocarcinoma in situ (AIS), grade 2 or 3 vulvar intraepithelial neoplasia (VIN2/3), and grade 2 or 3 vaginal intraepithelial neoplasia (VaIN2/3) [71]. The qHPV vaccine also provided effective and durable protection again lowgrade CIN, VIN, and VaIN [72] (Table 1).

In a phase III efficacy trial among men, qHPV also had high efficacy for prevention of genital warts among 4055 males aged 16 through 26 years. Among those who received all three vaccine doses and were seronegative at day 1 and DNAnegative at day 1 through month 7 to the respective HPV type, per-protocol efficacy for prevention of HPV 6-, 11-, 16-, and 18-related genital warts was 89.3\% (95\% CI=65.3-97.9\%); efficacy for HPV 6- and 11-related genital warts was similar. Efficacy for prevention of HPV 6-, 11-, 16- and 18-related genital warts among males who received at least one vaccine dose, regardless of baseline infection or serology, was $68.1 \%$ (95\% CI $=48.8-80.7 \%$ ) [76]. There was no clear evidence of protection from disease caused by HPV types for which boys and men were DNA-positive regardless of serostatus at baseline [76].

A sub-study of the phase III efficacy trial included 602 healthy men who have sex with men, 16 to 26 years of age, to receive either qHPV vaccine or placebo [74••]. Efficacy of the qHPV vaccine against AIN associated with HPV-6, 11, 16, or 18 was $50.3 \%(95 \% \mathrm{CI}=25.7-67.2)$ in the intention-totreat population and $77.5 \%(95 \% \mathrm{CI}=39.6-93.3)$ in the perprotocol efficacy population; the corresponding efficacies against AIN associated with HPV of any type were $25.7 \%$ (95\% CI $=-1.1-45.6)$ and $54.9 \%(95 \% \mathrm{CI}=8.4-79.1)$, respectively. The rate of AIN2/3 related to infection with HPV$6,11,16$, or 18 was reduced by $54.2 \%(95 \% \mathrm{CI}=18.0-75.3)$ in the intention-to-treat population and by $74.9 \%(95 \% \mathrm{CI}=$ 8.8-95.4) in the per-protocol efficacy population. The corresponding risks of persistent anal infection with HPV-6, 11, 16, or 18 were reduced by $59.4 \%(95 \% \mathrm{CI}=43.0-71.4)$ and $94.9 \%(95 \% \mathrm{CI}=80.4-99.4)$, respectively. At this time, the Advisory Committee on Immunization Practices (ACIP) recommends routine vaccination with bivalent or $\mathrm{qHPV}$ vaccine for females aged 11 or 12 years and with qHPV vaccine for males aged 11 or 12 years. Vaccination also is recommended for females aged 13-26 years and for males aged 13-21 years who were not vaccinated previously. MSM may be vaccinated up to 26 years of age.

The burden of HPV-associated disease has been abundantly demonstrated in people with HIV/AIDS. While the secondary prevention strategy for cervical and anal cancer has doubtlessly saved lives, primary prevention of HPV infection in this population is critically needed. Two separate AIDS Malignancy Consortium trials evaluated the safety and immunogenicity of qHPV vaccine in HIV-infected men and women. In HIVinfected men and women, seroconversion rates for HPV types contained in the vaccine were well over $80 \%$ [77, 78•]. No adverse effects on CD4 counts and plasma HIV-1 RNA levels were observed [77]. Women with HIV RNA load $>10$, 000 copies/mL and/or CD4 count $<200$ cells/ $\mu \mathrm{L}$ had lower 
Table 1 Efficacy of approved HPV vaccines against genital lesions

\begin{tabular}{|c|c|c|c|}
\hline Neoplasia grade & Vaccinated/infected $(n)$ & Control vaccinated/infected $(n)$ & $\begin{array}{l}\text { Vaccine efficacy } \\
(95 \% \mathrm{CI})\end{array}$ \\
\hline \multicolumn{4}{|c|}{ CIN1 in baseline-negative cohort [73] } \\
\hline CIN1 & $4616 / 241$ & $4680 / 346$ & $29.7 \%(17-41 \%)$ \\
\hline CIN1 (HPV 16,18) & $4.616 / 3$ & $4680 / 107$ & $97.2 \%(92-99 \%)$ \\
\hline \multicolumn{4}{|c|}{ CIN1 in intention-to-treat cohort [73] } \\
\hline CIN1 & $8562 / 788$ & $8598 / 984$ & $20.3 \%(12-28 \%)$ \\
\hline CIN1 (HPV 16, 18) & $8562 / 99$ & $8598 / 304$ & $67.5 \%(59-74 \%)$ \\
\hline \multicolumn{4}{|c|}{ CIN2+ in baseline-negative cohort [73] } \\
\hline CIN2+ & $4616 / 77$ & $4680 / 136$ & $42.7 \%(24-57 \%)$ \\
\hline CIN2+ (HPV 16, 18) & $4616 / 0$ & $4680 / 45$ & $100 \%(89-100 \%)$ \\
\hline \multicolumn{4}{|c|}{ CIN2+ in intention-to-treat cohort [73] } \\
\hline CIN2+ & $8562 / 296$ & $8598 / 367$ & $19.3 \%(6-31 \%)$ \\
\hline CIN2+ (HPV 16, 18) & $8562 / 79$ & $8598 / 168$ & $53 \%(36-60 \%)$ \\
\hline \multicolumn{4}{|c|}{ CIN3 in baseline-negative cohort [73] } \\
\hline CIN3 & $4616 / 36$ & $4680 / 64$ & $43 \%(13-63 \%)$ \\
\hline CIN3 (HPV16, 18) & $4616 / 0$ & $4680 / 34$ & $97.2 \%(92-99 \%)$ \\
\hline \multicolumn{4}{|c|}{ CIN3 in intention-to-treat cohort [73] } \\
\hline CIN3 & $8562 / 237$ & $8598 / 284$ & $16.4(0.4-30 \%)$ \\
\hline CIN3 (HPV 16, 18) & $8562 / 100$ & $8598 / 177$ & $43.5 \%(27-56 \%)$ \\
\hline \multicolumn{4}{|c|}{ AIN in baseline-negative cohort $[74 \bullet \cdot]$} \\
\hline Persistent HPV 16, 18 & $191 / 6$ & $205 / 39$ & $84.5 \%(63-95 \%)$ \\
\hline AIN1+ (men) & $129 / 12$ & $126 / 28$ & $54.9 \%(8-79 \%)$ \\
\hline AIN2-3 (men) & $194 / 3$ & $208 / 13$ & $74.9 \%(9-95 \%)$ \\
\hline \multicolumn{4}{|c|}{ AIN intention-to-treat cohort $[74 \bullet \bullet]$} \\
\hline Persistent HPV 16, 18 & $275 / 52$ & $276 / 92$ & $46.5 \%(24-63 \%)$ \\
\hline $\mathrm{AIN} 1+(\mathrm{men})$ & $275 / 74$ & $276 / 103$ & $25.7 \%(-1.1-46 \%)$ \\
\hline AIN2-3 (men) & $275 / 18$ & $276 / 48$ & $54.2 \%(18-75 \%)$ \\
\hline \multicolumn{4}{|c|}{ External genital warts in baseline-negative women [72] } \\
\hline HPV 6, 11 & $4689 / 4$ & $4735 / 138$ & $97.1 \%(92-99 \%)$ \\
\hline \multicolumn{4}{|c|}{ External genital warts in intention-to-treat women [72] } \\
\hline HPV 6, 11 & $8689 / 62$ & $8702 / 298$ & $79.3 \%(73-85 \%)$ \\
\hline \multicolumn{4}{|c|}{ External genital warts in baseline-negative men [75] } \\
\hline HPV 6 & $1245 / 3$ & $1244 / 19$ & $84.3 \%(47-97 \%)$ \\
\hline HPV 11 & $1245 / 1$ & $1244 / 11$ & $90.9 \%(38-100 \%)$ \\
\hline \multicolumn{4}{|c|}{ External genital warts in intention-to-treat men [75] } \\
\hline HPV 6 & $1817 / 63$ & $1815 / 112$ & $44.7 \%(24-60 \%)$ \\
\hline HPV 11 & $1817 / 16$ & $1815 / 39$ & $59.4 \%(26-79 \%)$ \\
\hline
\end{tabular}

rates of seroconversion rates [78•]. Currently, ACIP and the World Health Organization recommend administration of HIV-infected women with either bivalent or qHPV vaccine through age 26 years for those who have not been vaccinated previously or who have not completed the three-dose series [79]. ACIP also recommends qHPV vaccination of HIVinfected men up to the age of 26 years.

While both the bivalent and qHPV vaccines have been shown to be safe and highly effective against vaccine-type persistent HPV infection and lesions, there is growing interest in preventing a wider range of HPV types beyond HPV 16 and
18 since HPV 16 and 18 cause only $70 \%$ of cervical cancers. Recent results suggest that the bivalent vaccine may confer some cross-protection against high-risk HPV types -31, -33, -45, -52, and -58 [69, 80-82]. In December 2014, the US FDA approved Merck's nonavalent HPV vaccine (Gardasil 9). In addition to HPV-16/18, the nonavalent vaccine also targets HPV types 31/33/ $45 / 52 / 58$. These seven HPV types cause approximately $50 \%$ of cases of CIN1, 85-90\% of HPV-related vulvar cancers, 80-85\% of HPV-related vaginal cancers, and 90-95\% of HPV-related anal cancers [52]. 
The nonavalent HPV vaccine was studied in six clinical trials including over 13,000 individuals aged 9-26 years. It was found to be safe, with efficacy of $93 \%$ or higher against anogenital lesions/cancers caused by the included HPV types [83]. At least $99.7 \%$ of individuals included in the analyses for each HPV type became seropositive for that type by month 7. Its effectiveness against persistent infection and disease related to HPV types $6,11,16$, or 18 was inferred from noninferiority comparisons in girls and women aged 9-26 of geometric mean titers (GMTs) following vaccination with the nonavalent vaccine with those following vaccination with qHPV [83]. The nonavalent vaccine prevented HPV 31-, 33-, 45-, 52-, and 58-related persistent infection and disease and also reduced the incidence of HPV 31-, 33-, 45-, 52-, and 58-related cytologic abnormalities, cervical and external genital lesions on biopsy, and definitive therapy. Effectiveness of the nonavalent vaccine against anal lesions was inferred from the efficacy of qHPV against anal lesions caused by HPV types $6,11,16$, and 18 in men and antibody responses elicited by the nonavalent vaccine against the HPV types covered by the vaccine. In addition, the safety profile of the nonavalent vaccine was similar in those who previously received the qHPV vaccine as in those who were naive to HPV vaccination [83].

The projected population-level effectiveness of the nonavalent HPV vaccine was evaluated by an individualbased, transmission-dynamic model of HPV infection and disease [84]. Switching to a nonavalent vaccine has the potential to further reduce precancerous lesions and cervical cancer. However, in men, it has been predicted that the nonavalent vaccine could only make incremental gains over the qHPV vaccine over 30 years. This is because most HPV-related cancers are caused by HPV-16/18 for which the first-generation HPV vaccines are highly effective against. The nonavalent vaccine has already been approved for girls and women aged 9-26 and boys 9-15 for prevention of warts, precancerous lesions, and cancer in the lower genital tract. It is currently under review by ACIP to determine how this vaccine will be implemented in the US population.

\section{Conclusion}

HPV is one of the most common STIs. The most important clinical consequence of HPV infection is cervical cancer. However, HPV-related cancers in men also cause significant morbidity and mortality. More research is required to realize better insight into the natural history of oral HPV infection. It is not effective or practical to routinely screen men and women for HPV. The three prophylactic HPV vaccines are proven to be highly efficacious against persistent cervical infections and against anal infections in the case of the qHPV vaccine. Although not demonstrated in randomized controlled trials, they may prevent oral HPV infection as well. The focus of organized HPV cancer prevention programs should include both reduction of the HPV disease burden among those already exposed to HPV and HPV vaccination to reduce the burden of high-risk HPV and low-risk HPV types who have not yet been exposed.

\section{Compliance with Ethics Guidelines}

Conflict of Interest C-CJ Wang declares no conflicts of interest.

JM Palefsky serves on the Scientific Advisory Board for Merck, Hera Therapeutics, and The Vax; has received research grants from Merck and Hologic; has served as a consultant for Cel Sci; has received honorarium from Bristol-Meyers Squibb; and has received travel reimbursement from Merck and Hologic.

Human and Animal Rights and Informed Consent All studies by the authors involving animal and/or human subjects were performed after approval by the appropriate institutional review boards. When required, written informed consent was obtained from all participants.

\section{References}

Papers of particular interest, published recently, have been highlighted as:

- Of importance

•• Of major importance

1. Burchell AN, Tellier PP, Hanley J, et al. Human papillomavirus infections among couples in new sexual relationships. Epidemiology. 2010;21:31-7.

2. Nyitray AG, Menezes L, Lu B, et al. Genital human papillomavirus (HPV) concordance in heterosexual couples. J Infect Dis. 2012;206:202-11.

3. Burchell AN, Winer RL, de Sanjose S, et al. Chapter 6: epidemiology and transmission dynamics of genital HPV infection. Vaccine. 2006;24 Suppl 3:S3/52-61.

4. Giuliano AR, Lee JH, Fulp W, et al. Incidence and clearance of genital human papillomavirus infection in men (HIM): a cohort study. Lancet. 2011;377:932-40.

5. Giuliano AR, Lazcano E, Villa LL, et al. Circumcision and sexual behavior: factors independently associated with human papillomavirus detection among men in the HIM study. Int J Cancer. 2009;124:1251-7.

6. Burchell AN, Coutlee F, Tellier PP, et al. Genital transmission of human papillomavirus in recently formed heterosexual couples. J Infect Dis. 2011;204:1723-9.

7. Hoots BE, Palefsky JM, Pimenta JM, et al. Human papillomavirus type distribution in anal cancer and anal intraepithelial lesions. Int J Cancer. 2009;124:2375-83.

8. Ronco G, Arbyn M, Segnan N. Cervical screening according to age and HPV status. BMJ. 2009;339:b3005.

9. Moscicki AB, Widdice L, Ma Y, et al. Comparison of natural histories of human papillomavirus detected by clinician- and self-sampling. Int J Cancer. 2010;127:1882-92.

10. Schiffman M, Rodriguez AC, Chen Z, et al. A population-based prospective study of carcinogenic human papillomavirus variant lineages, viral persistence, and cervical neoplasia. Cancer Res. 2010;70:3159-69. 
11. Tota JE, Chevarie-Davis M, Richardson LA, et al. Epidemiology and burden of HPV infection and related diseases: implications for prevention strategies. Prev Med. 2011;53 Suppl 1:S12-21.

12. Chaturvedi AK, Engels EA, Pfeiffer RM, et al. Human papillomavirus and rising oropharyngeal cancer incidence in the United States. J Clin Oncol. 2011;29:4294-301.

13. Schiffman M, Wentzensen N, Wacholder S, et al. Human papillomavirus testing in the prevention of cervical cancer. J Natl Cancer Inst. 2011;103:368-83.

14. Silverberg MJ, Lau B, Justice AC, et al. Risk of anal cancer in HIVinfected and HIV-uninfected individuals in North America. Clin Infect Dis. 2012;54:1026-34.

15. Crum-Cianflone NF, Hullsiek KH, Marconi VC, et al. Anal cancers among HIV-infected persons: HAART is not slowing rising incidence. AIDS. 2010;24:535-43.

16. Palefsky JM. Anal cancer prevention in HIV-positive men and women. Curr Opin Oncol. 2009;21:433-8.

17. Thomas M, Pim D, Banks L. The role of the E6-p53 interaction in the molecular pathogenesis of HPV. Oncogene. 1999;18:7690-700.

18. Boyer SN, Wazer DE, Band V. E7 protein of human papilloma virus-16 induces degradation of retinoblastoma protein through the ubiquitin-proteasome pathway. Cancer Res. 1996;56:4620-4.

19. Klingelhutz AJ, Roman A. Cellular transformation by human papillomaviruses: lessons learned by comparing high- and low-risk viruses. Virology. 2012;424:77-98.

20. Doorbar J, Quint W, Banks L, et al. The biology and life-cycle of human papillomaviruses. Vaccine. 2012;30 Suppl 5:F55-70.

21.•Darragh TM, Colgan TJ, Thomas Cox J, et al. The Lower Anogenital Squamous Terminology Standardization project for HPV-associated lesions: background and consensus recommendations from the College of American Pathologists and the American Society for Colposcopy and Cervical Pathology. Int J Gynecol Pathol. 2013;32:76-115. This is an important update on terminology used to HPV-associated lesions in the lower genital tract.

22. Hariri S, Unger ER, Sternberg M, et al. Prevalence of genital human papillomavirus among females in the United States, the National Health And Nutrition Examination Survey, 2003-2006. J Infect Dis. 2011;204:566-73.

23. Winer RL, Lee SK, Hughes JP, et al. Genital human papillomavirus infection: incidence and risk factors in a cohort of female university students. Am J Epidemiol. 2003;157:218-26.

24. Marrazzo JM, Gorgos LM. Emerging sexual health issues among women who have sex with women. Curr Infect Dis Rep. 2012.

25. Winer RL, Hughes JP, Feng Q, et al. Early natural history of incident, type-specific human papillomavirus infections in newly sexually active young women. Cancer Epidemiol Biomarkers Prev. 2011;20:699-707.

26. Richardson H, Kelsall G, Tellier P, et al. The natural history of typespecific human papillomavirus infections in female university students. Cancer Epidemiol Biomarkers Prev. 2003;12:485-90.

27. Sundstrom K, Eloranta S, Sparen P, et al. Prospective study of human papillomavirus (HPV) types, HPV persistence, and risk of squamous cell carcinoma of the cervix. Cancer Epidemiol Biomarkers Prev. 2010;19:2469-78.

28. Schiffman M, Glass AG, Wentzensen N, et al. A long-term prospective study of type-specific human papillomavirus infection and risk of cervical neoplasia among 20,000 women in the Portland Kaiser Cohort Study. Cancer Epidemiol Biomarkers Prev. 2011;20:1398-409.

29. Koutsky LA, Holmes KK, Critchlow CW, et al. A cohort study of the risk of cervical intraepithelial neoplasia grade 2 or 3 in relation to papillomavirus infection. N Engl J Med. 1992;327:1272-8.

30. Bae J, Seo SS, Park YS, et al. Natural history of persistent high-risk human papillomavirus infections in Korean women. Gynecol Oncol. 2009;115:75-80.
31. Bory JP, Cucherousset J, Lorenzato M, et al. Recurrent human papillomavirus infection detected with the hybrid capture II assay selects women with normal cervical smears at risk for developing high grade cervical lesions: a longitudinal study of 3,091 women. Int J Cancer. 2002;102:519-25.

32. Cuzick J, Arbyn M, Sankaranarayanan R, et al. Overview of human papillomavirus-based and other novel options for cervical cancer screening in developed and developing countries. Vaccine. 2008;26 Suppl 10:K29-41.

33. Castle PE, Wacholder S, Lorincz AT, et al. A prospective study of high-grade cervical neoplasia risk among human papillomavirusinfected women. J Natl Cancer Inst. 2002;94:1406-14.

34. Kreimer AR, Bhatia RK, Messeguer AL, et al. Oral human papillomavirus in healthy individuals: a systematic review of the literature. Sex Transm Dis. 2010;37:386-91.

35. Gillison ML, Broutian T, Pickard RK, et al. Prevalence of oral HPV infection in the United States, 2009-2010. JAMA. 2012;307:693-703.

36. Chaturvedi AK, Anderson WF, Lortet-Tieulent J, et al. Worldwide trends in incidence rates for oral cavity and oropharyngeal cancers. J Clin Oncol. 2013;31:4550-9.

37. Mehanna H, Beech T, Nicholson T, et al. Prevalence of human papillomavirus in oropharyngeal and nonoropharyngeal head and neck cancer-systematic review and meta-analysis of trends by time and region. Head Neck. 2013;35:747-55.

38. Combes JD, Franceschi S. Role of human papillomavirus in nonoropharyngeal head and neck cancers. Oral Oncol. 2014;50:370-9.

39. de Pokomandy A, Rouleau D, Ghattas G, et al. Prevalence, clearance, and incidence of anal human papillomavirus infection in HIV-infected men: the HIPVIRG cohort study. J Infect Dis. 2009;199:965-73.

40. Vajdic CM, van Leeuwen MT, Jin F, et al. Anal human papillomavirus genotype diversity and co-infection in a community-based sample of homosexual men. Sex Transm Infect. 2009;85:330-5.

41. Hernandez AL, Efird JT, Holly EA, et al. Incidence of and risk factors for type-specific anal human papillomavirus infection among HIV-positive MSM. AIDS. 2014;28:1341-9. This is a good example of prospective cohort study on AIN in HIV-infected MSM.

42. Giuliano AR, Lazcano-Ponce E, Villa LL, et al. The human papillomavirus infection in men study: human papillomavirus prevalence and type distribution among men residing in Brazil, Mexico, and the United States. Cancer Epidemiol Biomarkers Prev. 2008;17:2036-43.

43. Chin-Hong PV, Vittinghoff E, Cranston RD, et al. Age-related prevalence of anal cancer precursors in homosexual men: the EXPLORE study. J Natl Cancer Inst. 2005;97:896-905.

44. Moreira Jr ED, Giuliano AR, Palefsky J, et al. Incidence, clearance, and disease progression of genital human papillomavirus infection in heterosexual men. J Infect Dis. 2014;210:192-9.

45. Donovan B, Franklin N, Guy R, et al. Quadrivalent human papillomavirus vaccination and trends in genital warts in Australia: analysis of national sentinel surveillance data. Lancet Infect Dis. 2011;11: 39-44. This study demonstrated a very interesting finding of herd immunity due to $H P V$ vaccination.

46. Shiels MS, Pfeiffer RM, Chaturvedi AK, et al. Impact of the HIV epidemic on the incidence rates of anal cancer in the United States. Journal of the National Cancer Institute; 2012;104(20):1591-8.

47. Roka S, Rasoul-Rockenschaub S, Roka J, et al. Prevalence of anal HPV infection in solid-organ transplant patients prior to immunosuppression. Transpl Int. 2004;17:366-9.

48. Patel HS, Silver AR, Northover JM. Anal cancer in renal transplant patients. Int J Colorectal Dis. 2007;22:1-5.

49. Tarallo PA, Smolowitz J, Carriero D, et al. Prevalence of high-risk human papilloma virus among women with hepatitis $\mathrm{C}$ virus before liver transplantation. Transpl Infect Dis. 2013;15:400-4.

50. Grulich AE, van Leeuwen MT, Falster MO, et al. Incidence of cancers in people with HIV/AIDS compared with immunosuppressed transplant recipients: a meta-analysis. Lancet. 2007;370:59-67. 
51. Strickler HD, Burk RD, Fazzari M, et al. Natural history and possible reactivation of human papillomavirus in human immunodeficiency virus-positive women. J Natl Cancer Inst. 2005;97:577-86.

52. Munoz N, Bosch FX, de Sanjose S, et al. Epidemiologic classification of human papillomavirus types associated with cervical cancer. N Engl J Med. 2003;348:518-27.

53. Massad LS, Fazzari MJ, Anastos K, et al. Outcomes after treatment of cervical intraepithelial neoplasia among women with HIV. J Low Genit Tract Dis. 2007;11:90-7.

54. Harris TG, Burk RD, Palefsky JM, et al. Incidence of cervical squamous intraepithelial lesions associated with HIV serostatus, CD4 cell counts, and human papillomavirus test results. JAMA. 2005;293:1471-6.

55. Xue X, Gange SJ, Zhong Y, et al. Marginal and mixed-effects models in the analysis of human papillomavirus natural history data. Cancer Epidemiol Biomarkers Prev. 2010;19:159-69.

56. Denny LA, Franceschi S, de Sanjose S, et al. Human papillomavirus, human immunodeficiency virus and immunosuppression. Vaccine. 2012;30 Suppl 5:F168-74.

57. Spinillo A, Debiaggi M, Zara F, et al. Human immunodeficiency virus type 1-related nucleic acids and papillomavirus DNA in cervicovaginal secretions of immunodeficiency virus-infected women. Obstet Gynecol. 2001;97:999-1004.

58. Huchko MJ, Woo V, Liegler T, et al. Is there an association between HIV-1 genital shedding and cervical intraepithelial neoplasia 2/3 among women on antiretroviral therapy? J Low Genit Tract Dis. 2013;17:354-60

59. Ghartey J, Kovacs A, Burk RD, et al. Genital tract HIV RNA levels and their associations with human papillomavirus infection and risk of cervical precancer. J Acquir Immune Defic Syndr. 2014;66:316-23.

60. Palefsky JM, Holly EA, Efirdc JT, et al. Anal intraepithelial neoplasia in the highly active antiretroviral therapy era among HIVpositive men who have sex with men. AIDS. 2005;19:1407-14.

61. Simard EP, Watson M, Saraiya M, et al. Trends in the occurrence of high-grade anal intraepithelial neoplasia in San Francisco: 20002009. Cancer. 2013;119:3539-45.

62. Palefsky JM, Holly EA, Ralston ML, et al. Prevalence and risk factors for anal human papillomavirus infection in human immunodeficiency virus (HIV)-positive and high-risk HIV-negative women. J Infect Dis. 2001;183:383-91.

63. Kojic EM, Cu-Uvin S, Conley L, et al. Human papillomavirus infection and cytologic abnormalities of the anus and cervix among HIV-infected women in the study to understand the natural history of HIV/AIDS in the era of effective therapy (the SUN study). Sex Transm Dis. 2011;38:253-9. This is one of the larger studies on HPV/AIN in HIV-infected women.

64. Hessol NA, Holly EA, Efird JT, et al. Concomitant anal and cervical human papillomavirusV infections and intraepithelial neoplasia in HIV-infected and uninfected women. AIDS. 2013;27:1743-51.

65. Goldie SJ, Kuntz KM, Weinstein MC, et al. The clinical effectiveness and cost-effectiveness of screening for anal squamous intraepithelial lesions in homosexual and bisexual HIV-positive men. JAMA. 1999;281:1822-9.

66. Dochez C, Bogers JJ, Verhelst R, et al. HPV vaccines to prevent cervical cancer and genital warts: an update. Vaccine. 2014;32: 1595-601.

67. Centers for Disease C, Prevention. FDA licensure of bivalent human papillomavirus vaccine (HPV2, Cervarix) for use in females and updated HPV vaccination recommendations from the Advisory Committee on Immunization Practices (ACIP). MMWR Morb Mortal Wkly Rep. 2010;59:626-9.

68. Lehtinen M, Paavonen J, Wheeler CM, et al. Overall efficacy of HPV-16/18 AS04-adjuvanted vaccine against grade 3 or greater cervical intraepithelial neoplasia: 4-year end-of-study analysis of the randomised, double-blind PATRICIA trial. Lancet Oncol. 2012;13:89-99.

69. Paavonen J, Naud P, Salmeron J, et al. Efficacy of human papillomavirus (HPV)-16/18 AS04-adjuvanted vaccine against cervical infection and precancer caused by oncogenic HPV types (PATRICIA): final analysis of a double-blind, randomised study in young women. Lancet. 2009;374:301-14.

70. Hildesheim A, Herrero R, Wacholder S, et al. Effect of human papillomavirus 16/18 L1 viruslike particle vaccine among young women with preexisting infection: a randomized trial. JAMA. 2007;298:743-53.

71. Kjaer SK, Sigurdsson K, Iversen OE, et al. A pooled analysis of continued prophylactic efficacy of quadrivalent human papillomavirus (types 6/11/16/18) vaccine against high-grade cervical and external genital lesions. Cancer Prev Res (Phila). 2009;2:868-78.

72. Group FIIS, Dillner J, Kjaer SK, et al. Four year efficacy of prophylactic human papillomavirus quadrivalent vaccine against low grade cervical, vulvar, and vaginal intraepithelial neoplasia and anogenital warts: randomised controlled trial. BMJ. 2010;341:c3493.

73. Munoz N, Kjaer SK, Sigurdsson K, et al. Impact of human papillomavirus (HPV)-6/11/16/18 vaccine on all HPV-associated genital diseases in young women. J Natl Cancer Inst. 2010;102:325-39.

74.• Palefsky JM, Giuliano AR, Goldstone S, et al. HPV vaccine against anal HPV infection and anal intraepithelial neoplasia. N Engl J Med. 2011;365:1576-85. This is the definitive study on role of HPV vaccine in prevention of AIN.

75. Giuliano AR, Palefsky JM, Goldstone S, et al. Efficacy of quadrivalent HPV vaccine against HPV Infection and disease in males. N Engl J Med. 2011;364:401-11.

76. Food and Drug Administration. Highlights of prescribing information. Gardasil (human papillomavirus quadrivalent [types 6, 11, 16 and 18]). Silver Spring, MD: Food and Drug Administration; 2011. Available at http:/www.fda.gov/downloads/biologicsbloodvaccines/vaccines/ approvedproducts/ucm111263.pdf. Accessed October 15, 2014.

77. Wilkin T, Lee JY, Lensing SY, et al. Safety and immunogenicity of the quadrivalent human papillomavirus vaccine in HIV-1-infected men. J Infect Dis. 2010;202:1246-53.

78. Kojic EM, Kang M, Cespedes MS, et al: Immunogenicity and safety of the quadrivalent human papillomavirus vaccine in HIV-1-infected women. Clin Infect Dis. 2014. This is the AIDS Malignancy Consortium study on HPV vaccination for HIV-infected women.

79. Markowitz LE, Dunne EF, Saraiya M, et al. Human papillomavirus vaccination: recommendations of the Advisory Committee on Immunization Practices (ACIP). MMWR Recomm Rep. 2014;63: $1-30$.

80. Brown DR, Kjaer SK, Sigurdsson K, et al. The impact of quadrivalent human papillomavirus (HPV; types 6, 11, 16, and 18) L1 virus-like particle vaccine on infection and disease due to oncogenic nonvaccine HPV types in generally HPV-naive women aged 16-26 years. J Infect Dis. 2009;199:926-35.

81. Olsson SE, Villa LL, Costa RL, et al. Induction of immune memory following administration of a prophylactic quadrivalent human papillomavirus (HPV) types 6/11/16/18 L1 virus-like particle (VLP) vaccine. Vaccine. 2007;25:4931-9.

82. David MP, Van Herck K, Hardt K, et al. Long-term persistence of anti-HPV-16 and -18 antibodies induced by vaccination with the AS04-adjuvanted cervical cancer vaccine: modeling of sustained antibody responses. Gynecol Oncol. 2009;115:S1-6.

83. Gardasil 9 prescribing information. Accessed on January 19, 2015. http://www.merck.com/product/usa/pi_circulars/g/gardasil_9/ gardasil_9_pi.pdf.

84. Van de Velde N, Boily MC, Drolet M, et al. Population-level impact of the bivalent, quadrivalent, and nonavalent human papillomavirus vaccines: a model-based analysis. J Natl Cancer Inst. 2012;104:1712-23. 